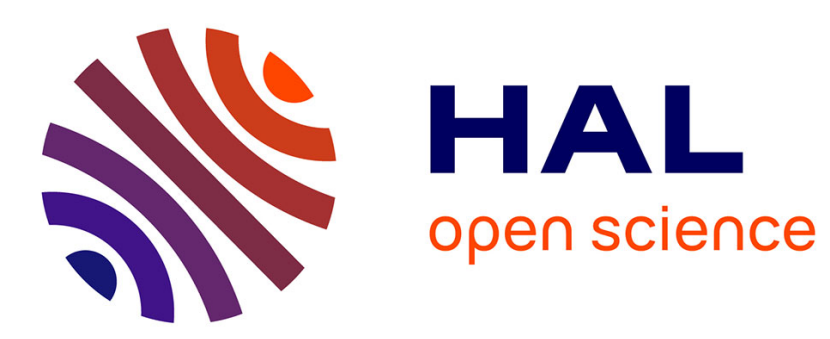

\title{
Dispositif expérimental d'étude de l'effet Faraday à basse température
}

\author{
A.C. Boccara
}

\section{To cite this version:}

A.C. Boccara. Dispositif expérimental d'étude de l'effet Faraday à basse température. Revue de Physique Appliquée, 1967, 2 (2), pp.142-144. 10.1051/rphysap:0196700202014201 . jpa-00242776

\section{HAL Id: jpa-00242776 https://hal.science/jpa-00242776}

Submitted on 1 Jan 1967

HAL is a multi-disciplinary open access archive for the deposit and dissemination of scientific research documents, whether they are published or not. The documents may come from teaching and research institutions in France or abroad, or from public or private research centers.
L'archive ouverte pluridisciplinaire HAL, est destinée au dépôt et à la diffusion de documents scientifiques de niveau recherche, publiés ou non, émanant des établissements d'enseignement et de recherche français ou étrangers, des laboratoires publics ou privés. 
remercier nos collègues physiciens des Laboratoires de Synthèse Atomique d'Ivry et de Physique Nucléaire d'Orsay pour leurs conseils précieux, et en particulier
M. Buchet qui a pris une part active dans la conception initiale de ce dispositif expérimental.

Manuscrit reçu le 15 février 1967.

\title{
BIBLIOGRAPHIE
}

[1] LEA (D. E.), Action of Radiations on Living cells, Cambridge Univ. Press, New York, 1946.

[2] ALLEN (A. O.), The radiation Chemistry of water and aqueous solutions, Van Nostrand Cie, Princeton, 1961.

[3] Pucheauli (J.), dans "Actions chimiques et biologiques des radiations ", $5^{\mathrm{e}}$ série, coll. dirigée par M. Haïssinsky, Masson, Paris, 1961.
[4] HaRT (E. J.), RAMber (W. J.) et RokiIN (S. R.), Rad. Res., 1956, 4, 378.

[5] Draganic (I. G.), Simic (M.), Mrlasin (N.) et RADAK (B.), Trudy Vsesaj. Sovesh. Rad. Khim., 64, Moscou, Izd. AN. SSSR., 1958.

[6] Whaling (W.), Encyclopedia of physics, vol. XXXIV, p. 208, Flugge, Springer-Verlag, Berlin, 1958.

[7] Pucheauli (J.) et Julien (R.), J. Chim. Phys. (en préparation).

\section{DISPOSITIF EXPÉRIMENTAL D'ÉTUDE DE L'EFFET FARADAY A BASSE TEMPÉRATURE}

\author{
Par A. G. BOCGARA, \\ Équipe de Recherche C.N.R.S. du Laboratoire d'Optique Physique, \\ École Supérieure de Physique et de Chimie, Paris.
}

\begin{abstract}
Résumé. - On décrit un montage permettant l'étude de l'effet Faraday à basse température. Le champ magnétique est produit par un petit aimant permanent, à pièces polaires percées, qui se trouve dans la queue du cryostat.

On donne les courbes de dispersion rotatoire magnétique d'un cristal de $\mathrm{CaF}_{2}$ dopé au $\mathrm{N}_{\mathrm{d}}^{3+}$ à différentes températures.

Abstract. - An apparatus is described for the study of the Faraday effect at low temperature. The magnetic field is produced by a small permanent magnet with bored pole pieces, which stands in the tail end of the cryostat.

Curves for the magnetic rotatory power of $\mathrm{N}_{\mathrm{d}}^{3+}$ in $\mathrm{CaF}_{2}$ are given for various temperatures.
\end{abstract}

L'intérêt que présente la mesure des effets magnétooptiques (pouvoir rotatoire et dichroïsme circulaire magnétiques) à basse température, dans le cas de cristaux dopés par exemple, nous a conduit à rechercher les meilleures conditions d'utilisation des spectromètres.

En effet, dans le domaine spectral (2 400-6 500 A) étudié, les conditions expérimentales (flux lumineux disponible, amplitude des phénomènes à mesurer, etc.) imposent l'emploi du montage expérimental au voisinage de sa limite de sensibilité.

Compte tenu des performances actuelles des spectromètres de mesure du pouvoir rotatoire magnétique ou du dichroïsme circulaire $[1,2]$, le choix d'un générateur de champ magnétique conditionne assez largement les performances d'un montage d'étude de la dispersion de l'effet Faraday.
Choix d'un générateur de champ magnétique. Le rapport signal sur bruit sera utilisé comme facteur de qualité des appareils de mesure de l'effet Faraday.

Le signal est proportionnel à l'intensité du champ magnétique. On a donc intérêt à choisir un générateur de champ assez puissant.

Cependant, le rapport signal sur bruit est proportionnel à la racine carrée de l'étendue géométrique du montage optique [1], c'est-à-dire inversement proportionnel aux dimensions du spectromètre, le long du faisceau lumineux, et limite l'encombrement du générateur.

Nous avons renoncé à l'emploi d'un solénoïde supraconducteur coûteux et d'un maniement délicat.

Un électroaimant à pièces polaires percées, placé selon l'axe du faisceau lumineux, réduit notablement l'étendue géométrique du spectromètre; de plus, les 
dimensions de l'entrefer, qui contient la queue du cryostat, ne permettent pas d'obtenir une valeur du champ supérieure à 8000 gauss. L'utilisation d'un tel électroaimant a donc été écartée ; elle conduisait, en outre, à des phénomènes gênants : action du champ magnétique de fuite sur le photomultiplicateur et pouvoir rotatoire magnétique parasite dû à l'introduction des fenêtres du cryostat dans l'entrefer.

La sensibilité du montage $\left(10^{-3}\right.$ à $10^{-4}$ degrés en dispersion rotatoire) interdit de placer un électroaimant perpendiculairement à l'axe du spectromètre

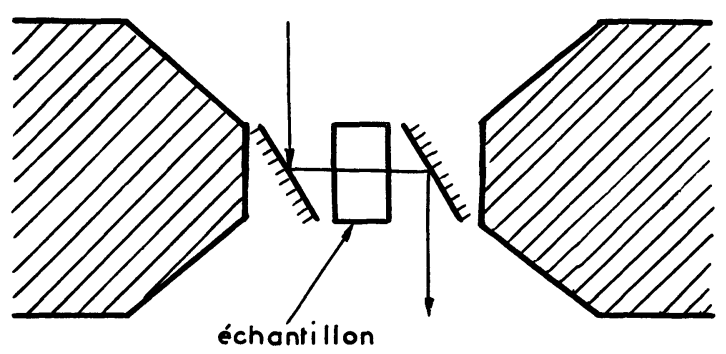

FIG. 1.

et de renvoyer les faisceaux à l'aide de petits miroirs ( fig. 1) : les effets optiques et magnéto-optiques sur les surfaces métalliques sont de l'ordre de grandeur des phénomènes étudiés.

Avec un petit aimant permanent à pièces polaires percées, on dispose d'un champ plus faible qu'avec un électroaimant (2600 à 4000 gauss), mais on évite certains des inconvénients cités précédemment.

En introduisant cet aimant dans la queue même du cryostat, on gagne un facteur important sur l'étendue géométrique du spectromètre et le rapport signal sur bruit est finalement comparable à celui que l'on obtient avec un électroaimant usuel.

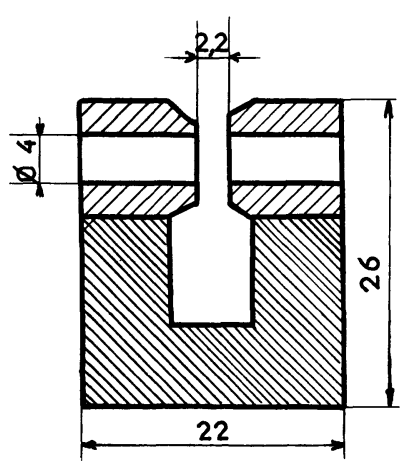

Montage avec 1 aimant Poids : $75 \mathrm{~g}$ Champ : 2600 gauss Dimensions : $26 \times 24 \times 22$

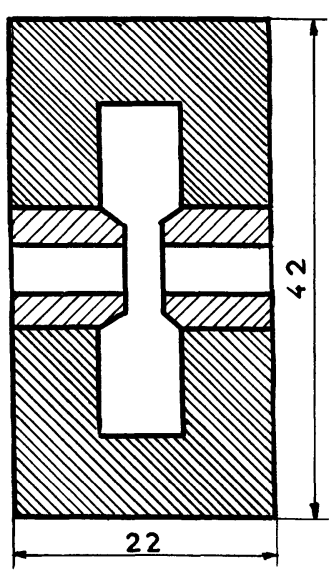

Montage avec 2 aimants Poids : $130 \mathrm{~g}$

Champ : 3900 gauss

Dimensions : $42 \times 24 \times 22$
Montage expérimental. a) Aimants. - On a réalisé deux générateurs de champ à partir des aimants Giffrey-Prêtre A. $3076 \mathrm{M}$ ( fig. 2).

b) Montage de L'aimant dans le cryostat ( fig. 3). - L'aimant est brasé sur la queue du cryostat avec un alliage fusible à $100^{\circ} \mathrm{C}$.

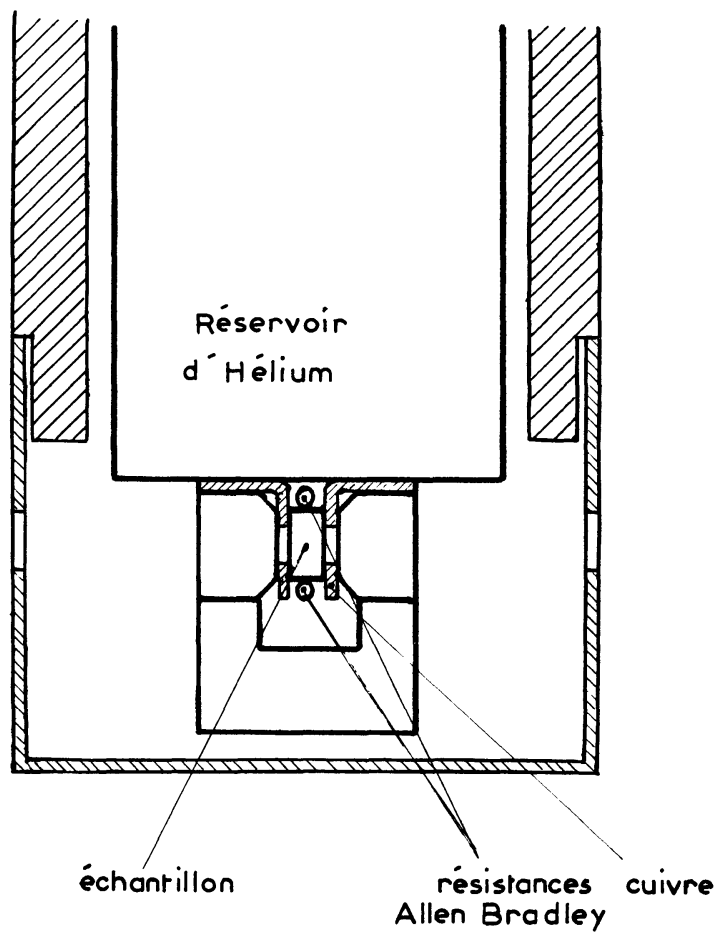

FIG. 3.

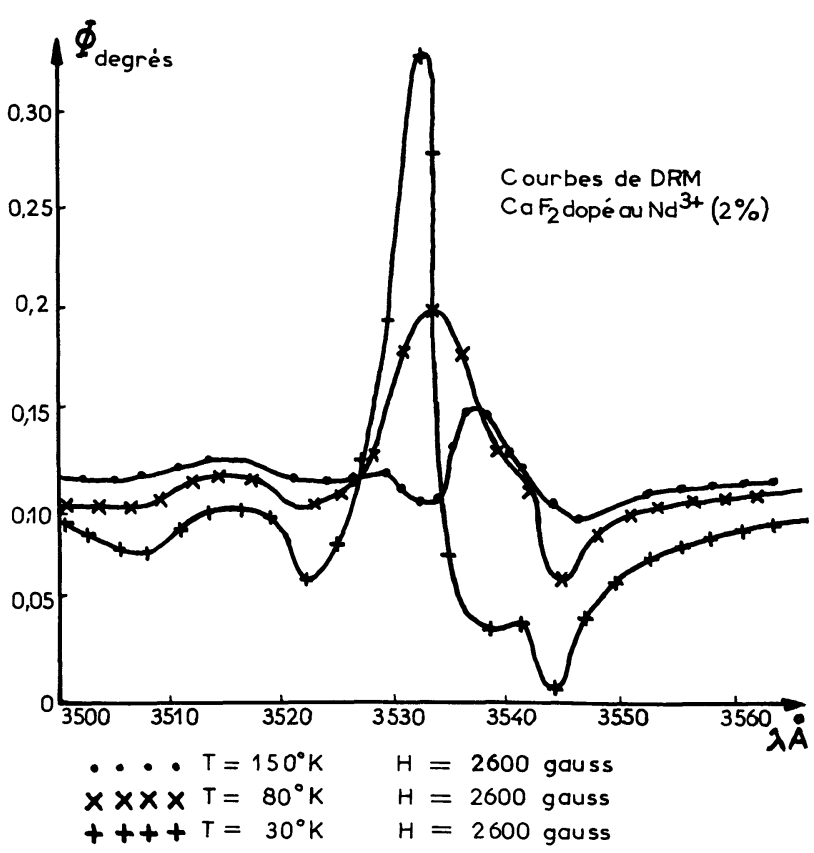

FIG. 4.

FIG. 2. 
L'échantillon est refroidi par deux lamelles de cuivre soudées sur le réservoir central ( $f g .3$ ).

c) Avec cet appareil, nous avons étudié la dispersion rotatoire magnétique d'un cristal de fluorine dopée au néodyme ( fig. 4). En utilisant différents fluides cryogéniques et en faisant varier la résistance thermique entre le réservoir central du cryostat et l'échantillon, nous avons $\mathrm{pu}$ obtenir les températures : $150 \mathrm{~K}$, $80^{\circ} \mathrm{K}, 30^{\circ} \mathrm{K}$ et $15 \circ \mathrm{K}$. Les températures sont mesurées à l'aide de deux résistances Allen Bradley collées sur l'échantillon ( $f g .3)$. On a vérifié ainsi que la température est homogène à mieux que $0,2 \mathrm{oK}$.

Remarques. - 1) On a constaté à l'aide d'un montage annexe que le champ magnétique ne variait pas de la température ambiante à celle de l'azote liquide.

2) Une bonne homogénéité du champ a été recherchée en étudiant la forme des pièces polaires à la cuve rhéographique. Une étude du champ avec une sonde à effet Hall de petite dimension a confirmé les prévisions.

Conclusion. - Les principaux avantages du dispositif employé sont les suivants :

$1^{0}$ Mise en place d'un générateur de champ fixe (2 600 à 4000 gauss) sans transformation du cryostat et à peu de frais.

$2^{\circ}$ Montage du cryostat sur un banc de mesure de l'effet Faraday fonctionnant à température ambiante, sans modification de son étendue géométrique.

Notons cependant que, à la température de l'hélium liquide, le refroidissement d'un tel ensemble par conduction n'est pas aisé, aussi nous appliquons-nous à améliorer le contact thermique entre l'échantillon et le réservoir d'hélium.

Manuscrit reçu le 23 janvier 1967.

\section{BIBLIOGRAPHIE}

[1] BADOZ (J.), BRIAT (B.), BILLARDon (M.), Design and evaluation of a new photoelectric spectropolarimeter for the study of Faraday effect dispersion, Jap. Jour. of App. Phys., 1965, 4, Sup. 1, 600.
[2] Birlatirdon (M.), BAdoz (J.), Mesure du dichroïsme circulaire dans le visible et l'ultraviolet, $C . R$. Acad. Sc., 1966, 263 B, 139. 